\title{
Islam, laïcité et la question d'autorité de l'Empire ottoman à la Turquie kémaliste
}

\section{Hamit Bozarslan}

\section{(2) OpenEdition \\ 1 Journals}

Édition électronique

URL : http://journals.openedition.org/assr/1036

DOI : 10.4000/assr.1036

ISSN : $1777-5825$

Éditeur

Éditions de l'EHESS

\section{Édition imprimée}

Date de publication : 1 janvier 2004

Pagination : 99-113

ISBN : 2-222-96741-4

ISSN : 0335-5985

\section{Référence électronique}

Hamit Bozarslan, «Islam, laïcité et la question d'autorité de l'Empire ottoman à la Turquie kémaliste », Archives de sciences sociales des religions [En ligne], 125 | janvier - mars 2004, mis en ligne le 22 février 2007, consulté le 19 avril 2019. URL : http://journals.openedition.org/assr/1036 ; DOI : 10.4000/ assr.1036 


\section{ISLAM, LAÏCITÉ ET LA QUESTION D'AUTORITÉ DE L'EMPIRE OTTOMAN À LA TURQUIE KÉMALISTE}

\section{Introduction}

Au premier abord, cet article paraîtra quelque peu à la marge du thème central de ce numéro spécial. Il porte non pas sur l'autorité religieuse en tant que telle, mais sur la religion et la laïcité comme sources de légitimation de l'autorité, et partant, du pouvoir politique. Ce choix, dicté par les conditions mêmes de la Turquie, montre les paradoxes du processus de sécularisation de ce pays, et au-delà, d'une grande partie du monde musulman. La sécularisation ne signifie pas, en l'occurrence, le retrait de l'autorité religieuse des affaires de l'ici-bas, mais bien sa constante mobilisation comme l'une des instances de légitimation du pouvoir politique auquel elle est subordonnée.

Comment s'étonner, dès lors, que le débat autour de la laïcité, qui fait rage dans ce pays depuis des décennies, demeure à plus d'un titre inintelligible : alors que l'islam se confond avec la nation et est présenté comme l'un des garants de son unité, voire de son unanimisme, la laïcité, mot fétiche qui résume toute la problématique de l'occidentalisation, s'avère être l'une des perpétuelles sources de sa division. Cette contradiction aboutit à une autre, bien plus violente : soumis ou non au pouvoir, le religieux est toujours conçu comme l'ennemi, réel ou potentiel, de la nation, laquelle ne peut pourtant exister sans la religion.

Quelle place accorder alors à l'autorité religieuse dans ce pays ? Il faut d'emblée souligner qu'ici - comme souvent ailleurs dans le monde musulman - les ulémas ne constituent plus la catégorie dite des hommes «qui lient et délient » (ahl al ahd wal hal). Alors que pendant de longs siècles, ils formaient une instance de contrôle et d'équilibre, chargée de limiter l'arbitraire du Prince dont ils acceptaient au préalable l'inévitabilité, ils ont désormais l'obligation d'obéir au pouvoir dont ils ne sont que de simples fonctionnaires (1). Ainsi, la présidence aux affaires

(1) Les hocas et les cheikhs des confréries, les dirigeants des communautés religieuses non-confrériques comme les Nurcus, Süleymanci ou encore Isikçi, ainsi que les responsables des courants culturels et/ou politiques islamistes font naturellement exception à cette règle. Mais leurs activités, tolérées ou réprimées selon les conditions politiques, sont théoriquement interdites par la loi et ils ne disposent d'aucune reconnaissance juridique. 
religieuses, attachée au premier ministre (2), s'affirme comme l'instance de tutelle de plus de 83000 commis d'État (3) habilités à exercer, au nom de l'autorité publique, la charge religieuse et de prononcer les hutbe (sermons) qui leur sont transmis depuis Ankara (4). Cette intégration du corps religieux à l'État, qui, comme nous le verrons plus loin, n'est que partiellement conforme à la matrice ottomane, est rarement remise en cause en Turquie : des plus islamistes aux athées, presque tous les acteurs de l'espace public admettent l'existence (ou du moins la nécessité) d'un «bon islam », authentique et tolérant, émanant de la «nation turque » et l'honorant en retour. Quoi de plus naturel, alors, que cet islam, «national» autant que « rationnel», "véritable rempart contre la discorde et l'ignorance » exige une organisation et une promotion étatiques ?

En Turquie, la ligne de fracture dans le domaine religieux ne passe donc pas entre les tenants et les opposants de l'intégration de l'islam à l'État, autrement dit, de l'exercice de l'autorité religieuse par le pouvoir politique lui-même ou de l'autonomisation mutuelle des champs religieux et politique. En revanche, on observe un véritable antagonisme, source d'une violence symbolique inouïe (5), entre les partisans et les opposants - tous deux, autoproclamés - de la laïcité érigée en pilier du régime républicain. Cette bataille, est-il nécessaire de le souligner, est dissociée de toute réflexion autour de la religion (6), mais aussi, voire surtout, de la nation. Comme la religion, la «nation turque » qui lui est consubstantielle, est acceptée comme une catégorie «naturelle», et donc hermétiquement fermée à toute remise en cause et à toute réinterprétation (7). La définition de la «nation » comme « famille », identifiable soit par l’islam, soit par la laïcité, soit, plus souvent, par les

(2) Dont la mission, redéfinie par la loi no 633 de 1965, a la charge « d'administrer les croyances, les pratiques et les principes moraux de la religion musulmane, éclairer la société à propos de la religion et gérer les lieux de culte ». Cité par Aydin UGur, "L'Ordalie de la démocratie en Turquie. Le Projet « communautarien islamique » d'Ali Bulaç et la laïcité », CEMOTI, no 19, 1995, p. 104.

(3) Ce chiffre n'inclut naturellement pas la haute bureaucratie de la Présidence des Affaires religieuses chargée de gérer les 66674 mosquées du pays. Cf. Jean-Paul Burdy, Jean MarCou, « Laïcité/ Laiklik : Introduction », CEMOTI, n 19,1995, p. 31. Le contraste est saisissant avec le cas français où « la République ne reconnaît, ne salarie ni ne subventionne aucun culte » (loi du 9.12.1905).

(4) Cf. pour l'organigramme de la Présidence des Affaires religieuses : http : //www.diyanet.gov.tr.

(5) Cf. par exemple : « Military Document: 'New War of Liberation Against Islamists », Turkish Daily News, 9.1.1999.

(6) La période allant du passage au pluralisme politique (1946) au coup d'État de 1980, témoigne cependant d'un débat riche autour de la religion. Durant ces décennies, l'islam gagne une visibilité inédite depuis la fondation de la République. Ce dynamisme, lu comme signe d'échec de la laïcité kémaliste, suscite l'intérêt de nombreux chercheurs. La fin de la mainmise d'un positivisme quasi religieux dans les universités turques, allant de pair avec la découverte, parfois dans un continuum éclectique, du marxisme et du wébérianisme, permet l'émergence d'une recherche qui voit dans la religion autre chose qu'une simple émanation de la « réaction contre-révolutionnaire » et de l'ignorance. Ainsi, pour la première fois depuis la fondation de la République, il devient possible d'analyser la religion en dehors des références internes du texte sacré, sensé conditionner le fait religieux, et de la syntaxe laïque, sensée fonder la société nouvelle. Dans une perspective fonctionnaliste, la religion est désormais analysée comme un concept et un fait polysémique et multifonctionnel, à partir des rôles contradictoires qui lui sont attribués : elle est perçue comme une arme des «opprimés » et/ou des "oppresseurs », se situant nécessairement dans une équation du pouvoir, qu'elle renforce ou qu'elle affaiblit. Dans un environnement intellectuel bien plus tendu, quelques études de très bon niveau, dépassant cette grille fonctionnaliste, ont vu le jour dans les années 1980 et 1990.

(7) Cf. entre autre, Étienne COPEAUX, Espaces et temps de la nation turque. Analyse d'une historiographie nationaliste (1931-1993), Paris, CNRS Éditions, 1997 ; Une vision turque du monde à travers les cartes de 1931 à nos jours, Paris, CNRS Éditions, 2000. 
deux, est acceptée comme un fait allant de soi, ne nécessitant aucune problématisation.

Cette série de non-dits, et donc de non-questionnés, qui crée une communion d'idées et d'esprits entre les partisans et les opposants de la laïcité kémaliste, tout en les opposant violemment par ailleurs, rend singulièrement complexe la tâche du chercheur. Celui-ci fait en effet face à un double objet de recherche, certes distinct, mais néanmoins inséparable. Il est invité, dans un premier temps, à déconstruire les doxa dominantes (kémaliste ou islamiste) sur la religion et la laïcité, et partant, la nation, troisième terme indissociable de l'équation. Dans un deuxième temps, cependant, il doit s'intéresser à la constitution du champ religieux, à son autonomie par rapport au pouvoir et/ou ses liens de subordination avec lui. Dans l'une et l'autre phases de cette démarche, il lui faudra être conscient du fait que les termes mêmes du débat, tels qu'ils sont dictés par les protagonistes (8), mais aussi, tels qu'ils sont élaborés par lui, contribuent à construire, ou du moins à façonner l'objet qu'il est sensé étudier.

Une approche qui s'inscrirait dans la sociologie historique, qui s'interrogerait, en même temps, sur l'évolution de la question religieuse et les multiples constructions politiques et idéologiques auxquelles elle donne lieu, pourrait apporter les instruments nécessaires pour surmonter ces multiples obstacles. Une telle lecture devrait prendre en considération la longue durée historique ainsi que les configurations qui ont provoqué des discontinuités et des ruptures au cours des deux derniers siècles.

Cet article tente de relever ce défi et prend délibérément ses distances par rapport à l'actualité brûlante des décennies 1980-1990 (9). Je formulerai d'abord quelques hypothèses sur les relations entre le pouvoir et la religion dans l'Empire ottoman avant les réformes de Tanzimat (1939). Dans un deuxième temps, j'analyserai les ruptures du $\mathrm{XIX}^{\mathrm{e}}$ siècle qui contribuent, à mon sens, à la formation du paysage politique et religieux de la future Turquie kémaliste. Enfin, dans la dernière partie de l'article, je suggérerai que la laïcité kémaliste, qui vise à interdire tout débat autour de la question religieuse, ne fait en réalité qu'approfondir les contradictions qui sont au fondement même de la République turque.

\section{L'Empire ottoman avant les Tanzimat}

La longue durée comprendra la période d'avant les réformes dites de Tanzimat, officiellement proclamées en 1839. Certes, ces longs siècles d'expansion puis de crises internes, de pertes de territoires et de déclin, ne peuvent être analysés à

(8) Rappelons les principaux termes qui marquent la syntaxe de l'establishment militaire, juridique et en partie universitaire en la matière : " progrès ", " réaction religieuse ", " courants réactionnaires ", «menaces réactionnaires », "mouvements contre-révolutionnaires ». Il est évident que ces concepts inventent ou diffusent un langage qui déborde le milieu de la recherche et participent à la définition du politique et du social sur la base du couple «ami-ennemi».

(9) Cf. sur ce sujet, Hamit Bozarslan, « Boire la coupe jusqu'à la lie : le Parti de la Prospérité en Turquie », Les travaux du Centre Marc Bloch, no 8, 1996, pp. 5-46. 
l'aune d'une seule dynamique. Une caractéristique leur donne un sens commun : durant ces siècles, les rapports entre le pouvoir et le religieux se voient codifiés aussi bien sur le plan doctrinal que par une praxis de pouvoir bien rôdée. La religion occupe une place centrale dans la définition et la légitimation du pouvoir, mais n'en constitue que l'un des piliers. On peut en effet ainsi résumer la doctrine ottomane de l'État: le pouvoir émane d'ici-bas et relève de l' " ordre du monde » (nizam-ul alem) aux sens propre et figuré du terme, mais celui-ci ne saurait être en contradiction avec la volonté divine. De même, l'obéissance au pouvoir, bien qu'émanant d'ici-bas, est un impératif religieux, dans la mesure où la communauté des musulmans (et au-delà, la communauté humaine) ne peut accepter la discorde (fitna). C'est à ce titre de garant de la concorde, et non à celui d'un quelconque ordre théocratique, que le Sultan-Khalife est considéré comme l'ombre de Dieu sur terre. La religion, en somme, qui marque de toute sa pesanteur les rituels de l'ordre social, et partant, du pouvoir, ne constitue donc pas leur essence ou leur finalité.

On peut dès lors légitimement parler d'une complémentarité entre les registres politique et religieux dans l'Empire d'avant les Tanzimat et non pas de la subordination du premier au deuxième. Certes, à sa formation, l'Etat ottoman doit beaucoup aux derviches colonisateurs qui véhiculent l'esprit de ghaza (expédition militaire contre les infidèles). Mais celui-ci est loin d'être le seul facteur qui assure son expansion; d'ailleurs d'autres groupes, parmi lesquels les non musulmans, acceptent la souveraineté du Sultan ottoman pour d'autres raisons et sans contrainte (10), ou la contestent pour des motifs qui n'ont rien à voir avec la religion. De même, même après la conquête de l'Égypte, qui permet en 1517 à Selim II de s'approprier le titre de khalife, les Ottomans ne justifient pas la guerre contre les kuffar (infidèles) simplement par une da'wa religieuse ou par le jihad. Pourquoi se présenter d'ailleurs comme un État banalement musulman, réduit à utiliser la seule doctrine du jihad, alors qu'on peut se revendiquer comme successeur des Romains et des Byzantins, comme la nouvelle incarnation de l'Imperium universel, autrement dit, comme la Troisième Rome ? Dans la continuité des États qui le précèdent, mais bien plus qu'eux, le " pouvoir » est en effet, en Ottoman, synonyme de devlet, mot polysémique signifiant autant "fortune tournante » qu'Imperium. La guerre dans cette perspective n'est pas, du moins exclusivement, une obligation découlant de la religion: elle fait partie des droits et des prérogatives de l'Imperium.

Comme le suggère en effet le sociologue Serif Mardin, dans l'équation ottomane de "din ve devlet» (religion et État) c'est bien le deuxième terme qui est déterminant (11). C'est aussi celui-ci qui autorise toute sorte de contournement de la sharia. Loin d'être contraire à la religion, la méthode de Hile-i seriye (12), qui consiste à respecter la lettre, mais pas l'esprit de la sharia, est légitimée aussi bien par la bureaucratie militaire et civile que par les ulemas. Elle signifie raison d'État avant la lettre. Cette pratique qui n'a pas peur de prononcer son nom, constitue une nécessité pour assurer la pérennité de l’Etat.

(10) Cf. notamment, Cemal Kafadar, Between Two Worlds: The Constitution of the Ottoman State, Berkeley-Los Angeles, University of California Press, 1995.

(11) Cf. notamment, le recueil de ses articles, Türkiye'de Din ve Siyaset. Makaleler III, Istanbul, Iletisim, 1991.

(12) Hila (hile, en turc) signifie : «'expédients juridiques', utilisés par certains juristes ou faqihs, surtout hanefites, pour modifier les règles des droits religieux ou fiqh», Dominique SourdeL, Janine Sourdel-Thomine, Vocabulaire de l'Islam, Paris, PUF, 2002, p. 46. 
Ainsi, en suivant l'argumentaire qu'Olivier Carré développe pour les États musulmans, on peut évoquer l'existence d'une sorte de sécularisme dans l'Empire ottoman, qui donne la priorité au pouvoir et à l'ici-bas (13). Cependant, comme dans d'autres sociétés pré-modernes (et nombre de sociétés modernes), ce sécularisme ne se définit pas en termes antinomiques avec la religion. L'État entretient des rapports complexes, fort fluides, à la fois d'autonomisation et de subordination, avec l'espace religieux. Ainsi, il admet l'existence d'un monde confrérique, qui échappe largement à son contrôle, ou avec lequel il entretient des relations de type clientélistes (nombre de bureaucrates de haut rang sont disciples des cheikhs de confréries). Il ne dispose pas moins cependant de son propre corps religieux, le seul qui soit officiellement reconnu comme instance habilitée à promulguer des fetvas et à rendre la justice. Sans doute parce que ces deux prérogatives, notamment la deuxième, s'avèrent irréductibles au domaine religieux proprement dit, que ce corps officiel est directement intégré au pouvoir, les ulémas étant assimilés à la classe dite militaire (askeri) (14). Cette intégration dote l'État des moyens de contrôler le champ religieux, sans pour autant marquer une quelconque évolution vers un système théocratique. De même, les ulemas eux-mêmes entretiennent des relations complexes avec le pouvoir. Ils ne constituent pas une simple instance subordonnée au Prince : comme les autres composantes de la bureaucratie ottomane, ils forment un élément de contrôle et d'équilibre interne au Palais. À ce titre, ils font partie du pouvoir et en limitent dans le même temps le champ de manœuvre (15).

Enfin, il importe de souligner que si la religion détermine l' «identité » du «Centre » ottoman, ni le «Centre », ni la «périphérie » (16) ne renvoient à une distinction en termes de communautés ethniques ou confessionnelles. Si, la non-adhésion à l'islam entraîne automatiquement un statut de subordonné (celui de zimmî, qui ne peut porter des armes et qui a l'obligation de payer la taxe de capitation), être musulman ne suffit nullement pour figurer au Centre. Les lignes de démarcation passent entre la mince strate de détenteurs du pouvoir et de multiples communautés et groupements, musulmans ou non, dont les dignitaires disposent des privilèges de "most favored lords ». Le "contrat tacite» ottoman (17) qui, sans écarter l'usage massif de la coercition, privilégie l'obéissance obtenue par la

(13) Olivier CARré, L'islam lä̈que ou le retour à la grande tradition, Paris, Armand Collin, 1993.

(14) Pour une lecture nuancée de la « fonctionnarisation » des ulémas, cf. Gilles VeINSTEIN, « Les Ottomans : fonctionnarisation des clercs, cléricalisation de l'État », in Dominique IogNA-Prat, Gilles Veinstein, Histoires des Hommes de Dieu dans l'islam et le christianisme, Paris, Flammarion, 2003, pp. 179-202.

(15) Comme le précise Kelsay, classiquement, «through the associated institutions of the masjid (mosque, place of communal gathering), the madrasa (religious school), and eventually jami'a (university), the 'ulema established a kind of sphere of influence, politically relevant but not quite "governmental", that limited the power of government officials. To my mind, this sphere represents the closest analogy in classical Islam to "civil society". John J. Kelsay, " Civil Society and Government in Islam » in Sohail H. Hasmi, ed., Islamic Political Ethics. Civil Society, Pluralism, and Conflict, PrincetonOxford, Princeton University Press, 2002, p. 10.

(16) Cf. Serif Mardin, "Center-Periphery Relations: A Key to Turkish politics », Daedalus, $\mathrm{n}^{\circ} 1$, vo102, 1973, pp. 169-190. Cf. aussi, Haim Gerber, « Ottoman Civil Society and Modern Turkish Democracy », in Kemal H. KarPat, dir., Ottoman Past and Today's Turkey, Leyde-Boston-Cologne, Brill, 2000, pp. 133-149.

(17) Pour ce concept, cf. Serif Mardin, Türk Modernlesmesi. Makaleler 4, Istanbul, Iletisim Yayinlari, 1991, p. 108. 
négociation, aboutit ainsi à une extrême fragmentation de la population ottomane, sur des critères de distinction religieuse (18), mais aussi linguistique ou simplement tribale.

\section{La période des crises ottomanes}

Ce rapide survol permet de conclure qu'à l'exception de certaines périodes (19), la religion ne constitue ni un enjeu central du pouvoir ni une source de tension majeure dans l'Empire ottoman. À partir de la deuxième moitié du $\mathrm{XIX}^{\mathrm{e}}$ siècle, cependant, elle devient un facteur de conflictualité à travers l'Empire ottoman, et ce, aussi bien au sein de la communauté sunnite dominante qu'entre les musulmans et les non-musulmans.

Il n'est pas nécessaire de revenir ici sur les multiples crises qui marquent ce long siècle qui voit la perte de confiance des Ottomans dans leur modèle, les pertes territoriales importantes, les pressions européennes et l'émergence des conflits communautaires, qui provoquent la première vague des nationalismes ottomans, ceux des populations chrétiennes, rapidement suivie de deux autres, celles des nationalismes des Turcs et des autres populations musulmanes (arabe, albanaise et kurde). Face à ces crises successives, annonciatrices du "déclin », l'Empire cherche à assurer son salut par et dans l'imitation de l'Occident. Mais le Palais a du mal à contrôler les effets de la politique d'occidentalisation dont il est le principal architecte. Celle-ci, en effet, pousse la nouvelle intelligentsia ottomane à s'identifier d'avantage aux «prophéties» scientifiques en vogue en Occident qu'à l'héritage ottoman ou à l'islam. La réponse du pouvoir à cette rupture générationnelle consiste à contrebalancer les effets pervers de l'occidentalisation par une politique de ré-islamisation. Contestée ou réappropriée, la religion devient ainsi la source de nouveaux espoirs, de tensions et de sens inédits.

Plusieurs registres, à commencer par l'évolution interne de l'islam sunnite, expliquent ces tensions qui apparaissent au cours de la lente décomposition de l'Empire. Dès la première moitié du XVIII' siècle, en effet, l'ordre ottoman est contesté de l'intérieur même de l'islam, notamment par le puritanisme wahabbite qui voit le jour en Arabie. Même localisé, celui-ci n'en ébranle pas moins le Palais ottoman, en le poussant à redéfinir ses positions doctrinales, et par conséquent, sa praxis du pouvoir. Cette première contestation est suivie par une deuxième, dite salafiste, qui, si du moins dans la lignée réformiste de Jamaladdin al-Afghani et Muhammad Abdou, ne découle pas directement du wahhabisme, ne montre pas moins l'ampleur du malaise parmi les musulmans de l'Empire, notamment au sein

(18) Cela est également le cas des «Apostats» (Alévis, Yézidis, Druzes) qui ont quitté 1'islam. S'ils sont théoriquement passibles de la mort, ils n'en sont pas moins intégrés dans le « contrat tacite ", qui leur assure des moyens de négociations directes ou indirectes avec le Palais. Pour l'une de ces communautés, les Yézidis, cf. Nelida Fuccaro, The Other Kurds. Yazidis in Colonial Iraq, Londres-New York, I.B. Tauris, 1999.

(19) Il y a cependant des périodes d'exception. Ainsi, lors des guerres ottomano-persanes du XVI siècle, diverses mouvances « hérétiques » ou messianiques qui s'inspirent du chi'isme persan, sont durement réprimées. 
de ses élites dans les régions périphériques. Comme le wahhabisme, mais peut-être, par moment à ses antipodes, le salafisme qui développe des critiques rigoureuses, et qui au XIX ${ }^{\mathrm{e}}$ siècle se propage bien au-delà des cercles religieux, pousse le Palais ottoman à apporter deux réponses contradictoires à la perte de confiance dans les anciens repères : se "ré-islamiser», chercher ainsi un surcroît de légitimité religieuse dont il n'avait pas besoin auparavant, et, simultanément, mettre l'accent sur la nature terrestre de son pouvoir, autrement dit, attester de la supériorité de l'ordre d'ici-bas sur celui de l'au-delà. De même, il se trouve dans l'obligation de s'inscrire dans un double projet contradictoire : se projeter comme puissance islamique à l'échelle planétaire et, en même temps, et en partant des critiques mêmes des salafistes qui soulignent l'affaiblissement du monde musulman, trouver les arguments légitimant l'« occidentalisation » (notamment, l'imitation de l'Occident, dans les domaines techniques et scientifiques). On trouve ici les prémisses d'une matrice qui va déterminer les rapports entre le pouvoir et la religion tout au long du $\mathrm{XIX}^{\mathrm{e}}$ siècle, voire du $\mathrm{XX}^{\mathrm{e}}$ siècle, sous la République. À chaque contestation venant de la sphère religieuse, le pouvoir sera ainsi obligé d'apporter une double réponse : affirmer la prééminence du terrestre, voire de la force pure et simple, mais aussi se ré-islamiser davantage, pour s'approprier le monopole de la référence religieuse et clientéliser et intégrer les confréries, notamment la nakchibandiyya (20). Cette dualité ottomane et turque, attise, plus qu'elle ne réduit, les contradictions liées aux rapports entre le pouvoir et la religion.

Enfin, parlant de l'intérieur de l'islam, un autre élément doit être pris en considération : tout au long du $\mathrm{XIX}^{\mathrm{e}}$ siècle, on assiste à une effervescence confrérique à travers l'ensemble de l'Empire ottoman. Or, par définition, les structures confrériques se prêtent mal à toute tentative de centralisation, de fonctionnarisation et de monopolisation par l'État, de la référence religieuse. Les rapports du pouvoir ottoman avec les milieux confrériques s'avèrent, d'ailleurs, à double tranchant : d'un côté, les confréries s'imposent comme sources potentielles de contestation (et nombre d'entre elles légitiment les révoltes qui secouent l'Empire durant ce siècle, voire y participent activement); de l'autre côté, par les liens de clientélisme qu'elles développent, elles permettent au pouvoir de disposer de relais à travers ses provinces, ce que sa bureaucratie totalement désorganisée, ne peut assurer. Mais une telle autonomie accordée aux confréries n'accroît pas moins la réaction qui émane des défenseurs de l'islam puritain, notamment de certains ulémas. Accusant les confréries d'ignorance, nombre d'entre eux glissent vers un radicalisme sans précédent (21).

Ainsi, au XIX $\mathrm{X}^{\mathrm{e}}$ siècle, l'équilibre «din ve devlet » (religion-pouvoir), qui assurait par le passé la primauté de l'ordre terrestre, mais qui, en même temps, le plaçait sous l'égide morale du céleste, est profondément ébranlé. Auparavant simple référence de légitimation du pouvoir et de l'ordre social, la religion est désormais chargée de nouvelles missions et de nouveaux sens. De même, elle se trouve dorénavant dans une nouvelle équation sécuritaire qui hypothèque son

(20) Cf. Ilber Ortayli, « The Policy of the Sublime-Porte towards Naqshbandis and Other Tariqas During the Tanzimat Period ", in Elisabeth Özdalga, dir., Naqshbandis in Western and Central Asia, Change and Continuity, Istanbul, Swedish Research Institute, 1999, pp. 67-72.

(21) Ainsi, Ubeydullah Efendi justifie ouvertement l'idée du régicide. Cf. Ahmet T. Alkan, Siradisi Bir Jön-Türk. Ubeydullah Efendi'nin Amerika Hatiralari, Istanbul, Iletisim, 1989, p. 62. Cf. aussi, Mümtazer TürkönE, Siyasi Ideoloji Olarak Islâmciligin Dogusu, Istanbul, Iletisim Yayinlari, 1991. 
avenir. Conscient de la nécessité de transformer l'Anatolie en bastion ultime de l'Empire, le Sultan Abdul Hamid II (1876-1908), se voit en effet obligé de constituer un sanctuaire musulman autour de cette contrée. Cette double construction nécessite un jeu complexe de légitimation. Ainsi, alors qu'il accentue le processus d'occidentalisation de son Empire, le seul selon lui capable d'en enrayer le déclin, il officialise, en même temps, le pan-islamisme comme la nouvelle doctrine officielle de son Palais.

Mais les tensions liées au domaine religieux ne se limitent pas à ces transformations internes à l'islam. Tout au long du siècle, en effet, les rapports entre les différentes communautés religieuses ottomanes se dégradent.

Pour comprendre cette détérioration, il convient de remonter aux vastes réformes, programmées depuis le règne de Selim III (1789-1807), mais réellement mises en chantier sous Abdul Madjid I ${ }^{\text {er }}$ à partir de 1839 et à leurs conséquences inattendues. Connues sous l'appellation générique des Tanzimat (réorganisations), celles-ci visent certes à établir l'égalité juridique entre les musulmans et les non-musulmans. Dans les faits, cependant, elles aboutissent à la transformation de la religion en un enjeu politique, voire à une nouvelle vision du monde dans laquelle les conflits et les rapports de domination et de forces entre les communautés musulmanes et non musulmanes, entre l'Empire ottoman et l'Occident, sont interprétés à partir d'une grille de lecture confessionnelle.

Il s'agit là d'une nouveauté qui réifie la religion. Avant le XIX ${ }^{\mathrm{e}}$ siècle, en effet, les Ottomans analysaient aussi bien la victoire que la déroute militaire à partir des purs rapports de forces entre eux et les autres puissances. Ces dernières étaient d'autant moins réduites à leur seul trait religieux que nombre d'entre elles figuraient parmi les alliées de la Sublime Porte, et ce contre leurs coreligionnaires. La religion n'était donc pas un référent identitaire, un critère normatif d'altérité ou encore le trait distinctif du zalim (oppresseur) et du mazlum (opprimé). Or, paradoxalement, au XIX ${ }^{\mathrm{e}}$ siècle, alors que l'Empire imite l'Occident pour assurer sa survie, les Ottomans lisent leurs rapports avec l'Occident à travers une grille de lecture de plus en plus confessionnelle et normative (22). Nombre de documents attestent en effet que le «nous » et les « autres » sont désormais définis à partir de la distinction religieuse, leurs rapports étant appréhendés comme révélateurs d'une lutte entre deux entités anhistoriques, voire, dans certains cas, simplement entre le bien et le mal. Il s'agit là en effet d'une double rupture épistémologique : alors que l'Occident chrétien qui, durant des siècles, associait le « Turc » à l'Antéchrist, le définit désormais comme l' " homme malade », qui, de surcroît, représente aussi les «ténèbres asiatiques », les Ottomans abandonnent l'interprétation fondée sur le nizam-ul alem, pour appréhender l'autre en tant qu'ennemi, parce que chrétien. Les termes comme progrès, civilisation, sciences... eux-mêmes ne gagnent de sens qu'à l'aune de cette double rupture (23). C'est à travers le contact avec la « civilisation» d' « autrui » que les Ottomans musulmans découvrent la «civilisation » comme concept et comme programme politique (24). C'est en se trouvant explicitement relégués au rang de "civilisation arriérée » qu'ils envisagent d'abord la possibilité,

(22) Cf. entre autres, les documents analysés par James J. ReID, Crisis of the Ottoman Empire. Prelude to Collapse 1839-1878, Stuttgart, Franz Steiner Verlag, 2001. 1990.

(23) Taha PARla, Babalar ve Ogullar. Tanzimat Romaninin Epistemolojisi, Istanbul, Iletisim Yayinlari,

(24) Cf. notamment, les articles de SaId-Halim Pasa, in Ertugrul DüZaG, ed., Buhranlarimiz ve Son Eserleri, Istanbul, Iz Yayincilik, 1991. 
puis l'«évidence » même, qu'ils ont également une civilisation, une civilisation moralement et spirituellement supérieure dont la religion constitue la clef de voûte. Les lignes de fractures communautaires et politiques qui, à travers les guerres successives (notamment de 1876-1877), se superposent à celles qui séparent les communautés ottomanes, ne font que renforcer cette perception.

En deuxième lieu, paradoxalement, avec le processus des Tanzimat, puis, la constitution de 1876 (25) qui instaure l'égalité de tous les Ottomans sans distinction confessionnelle ni linguistique, la religion devient un critère de classification des sujets ottomans. Certes, ces réformes assurent et codifient juridiquement l'égalité entre les musulmans et les non-musulmans. Mais plus que les pratiques et les coutumes du passé, la codification pose la question du statut de l'islam. Auparavant, les questions de la nature de l'État ottoman et du statut du sujet ottoman n'étaient pas formulées en termes juridiques, elles étaient posées par et dans une pratique informelle et évolutive. Or, avec l'adoption de la Constitution, la définition de soi comme entité étatique et comme sujet devient un enjeu à la fois symbolique et juridique, et les solutions trouvées s'avèrent tout aussi contraignantes que rigides. Avec l'adoption de la constitution de 1876, par exemple, l'islam devient la religion officielle de l'État, fixant par là même le critère d'appartenance à la majorité. De même, l'égalité entre les musulmans et les non-musulmans est constitutionnellement admise, mais elle est aussitôt équilibrée par la transformation des non-musulmans en communautés « minorisées », en opposition avec les musulmans dont la religion devient la religion d'État. Ainsi, les non-musulmans ne peuvent participer aux élections qu'en s'inscrivant sur des listes électorales qui leur sont réservées (26). Tout comme les projets de centralisation ottomane produisent plus de dé-centralité à travers l'Empire, les réformes garantissant l'accès à l'égalité juridique deviennent une source d'inégalité statutaire encore plus importante que par le passé.

Un facteur aggrave les effets de ces classifications : aussi bien les musulmans que les non-musulmans donnent une lecture supra-confessionnelle, hautement politique à ces transformations. Les réformes sont en effet introduites à un moment où les communautés ottomanes, surtout les non-musulmanes, se projettent comme nations, et aspirent, de plus en plus, non pas à une simple égalité avec les musulmans, mais à une scission d'avec l'Empire et à la constitution de leurs États nationaux. Auparavant unies avec les musulmans et ce, en dépit de leurs statuts inégaux, en tant que composantes des périphéries désireuses de garder une marge de manœuvre par rapport au Centre, elles constituent désormais les socles sociaux de nouvelles constructions politiques. La confession devient un critère important pour la définition de soi comme nation et, pour nombre de communautés chrétiennes, pour l'accès au statut d'État.

Or, ce passage de la communauté à la nation provoque d'emblée une tension entre la religion et la nation. Je ne mentionnerai ici qu'un exemple (27) : dans le contexte ottoman du XIX ${ }^{\mathrm{e}}$ siècle, d'un côté la nation ne peut trouver son trait

(25) Certes, cette constitution est suspendue trois mois après sa promulgation, pour être de nouveau mise en application en 1908. Mais par sa suspension (et non pas sa suppression pure et simple), elle devient la matrice juridique qui codifie le licite et l'illicite dans le domaine public.

(26) Taha ERdem, Anayasalar ve Seçim Kanunlari 1876-1982, Istanbul, Milliyet Yayinlari, 1982.

(27) Cf. Hamit Bozarslan, « Islam, nationalisme ou les paradoxes turcs », Après-Demain, no 447 , 2002, pp. 16-18. 
distinctif, sa mémoire historique, que dans une Église nationale, donc dans le registre religieux; de l'autre côté cependant, elle est menacée par ce même registre qui encourage les appartenances soit infra, soit supra-nationales qui, en tout état de cause, fixent des objectifs normatifs non-nationaux à la nation. Cette tension, qui interdit pourtant tout divorce entre la nation et la religion, continuera, entre autres dans certains pays balkaniques, jusqu'à la fin du $\mathrm{XX}^{\mathrm{e}}$ siècle.

La communauté musulmane se trouve, par mimétisme ou par contrainte, face aux mêmes dilemmes. Glissant de plus en plus de l'ottomanisme vers un mélange de turco-islamisme, puis clairement vers le nationalisme turc, le Centre ne peut concevoir la nation sans ce lien organique, intrinsèque, avec la religion. Influencé, comme les nationalismes des peuples chrétiens, à la fois par le romantisme et par le darwinisme social, il trouve dans la confession le socle, voire l'« essence » de la nation, dont la survie dans la guerre entre les espèces dépend du renforcement de soi en termes matériels et moraux. Ainsi, Ziya Gökalp (1876-1924), le penseur qui donne en gros sa formulation actuelle au nationalisme turc, lui fixe trois objectifs : islamisation, turcification et occidentalisation (28).

Mais quelle place accorder, dans cette nouvelle construction qu'est la nation, et dans cette nouvelle configuration qui implique à la fois le politique et l'espace public, autrement dit, tous les lieux de visibilité, à la religion ? D'une part, il semble évident qu'on ne peut concevoir, sous peine de la vider de toute spécificité, la nation sans religion; d'autre part, il apparaît tout aussi évident que la religion menace, potentiellement du moins, la même nation et risque de la vider de sa spécificité en tant qu'entité non confessionnelle. Ainsi, le courant islamiste qui émerge au lendemain du pronunciamiento de 1908 - qui rapidement, instaure le nationalisme turc comme la doctrine du nouveau régime unioniste -, formule ouvertement la revendication d'une unité musulmane contre tout nationalisme issu de peuples musulmans, dont le nationalisme turc. Pour nombre d'intellectuels kurdes et albanais, mais aussi turcs, le salafisme devient la syntaxe de l'unité musulmane, ouvertement anti-nationaliste. De même, si aucun islamiste ne demande l'instauration d'une théocratie, l'idée de la Sharia comme condensé de la civilisation musulmane, de la Constitution au sens juridique du terme, et de programme politique, n'en voit pas moins le jour. L'islamisme émerge ainsi dans le sillage du nationalisme avec lequel il a tout à la fois un rapport de complémentarité et d'hostilité. Mais en même temps que l'islamisme, émergent également les ulémas qui se conçoivent désormais comme classe-autorité, chargée de lire et de dire la loi, la sharia (29).

(28) Cf. Uriel Heyd, Foundations of Turkish Nationalism. The Life and Teachings of Ziya Gökalp, Londres, Lusac and Company, 1950 et Taha PARLA, The Social and Political Thought of Ziya Gökalp, 1876-1924, Leyde, E. J. Brill, 1985.

(29) Cf. les documents analysés par Ismail KARA, Islâmcilarin Siyasi Görüsleri, Istanbul, Iz Yayinlari, 1994, notamment p. 52. 


\section{Les contradictions kémalistes}

Ce lien paradoxal, de dépendance et de tension, entre la nation et la religion, est renforcé avec la fin de l'Empire ottoman et la proclamation, en 1923, de la République turque. Rappelons ici que l'extermination des Arméniens pendant la Première Guerre mondiale, les pogroms et la politique d'échange des populations chrétiennes contre les populations musulmanes (pas nécessairement turques) des Balkans aboutissent à une homogénéisation religieuse quasi-totale du territoire qu'allait devenir la Turquie actuelle (30) et constituent autant d'actes fondateurs de la «nation turque » (31). La Guerre d'Indépendance (1919-1922) contre les Arméniens et les Grecs et/ou contre les puissances victorieuses de la Première Guerre mondiale (Grande-Bretagne, France, Italie), qui se présente explicitement comme une guerre sainte, est menée au nom de la défense de l'islam et du khalifat (supprimé en 1924). $20 \%$ des députés de la Première Assemblée nationale, qui est l'instance d'autorité de cette guerre sont des ulémas (32). Ainsi, l'islam devient, au sens figuré comme au sens propre du terme, l'élément distinctif de la nouvelle carte d'identité et fixe le statut de la «nation turque ». La nouvelle umma qui émerge en Anatolie est délimitée par une territorialité nationale, qui se confond avec la « famille turque ». Cette nouvelle famille se constitue explicitement en opposition avec le reste du monde musulman et récuse, tout aussi ouvertement, l'héritage de l'Empire ottoman, considéré comme fossoyeur, durant des siècles, de la turcité. Il s'agit là d'une nouveauté et d'un paradoxe, car faire partie de la umma musulmane dans le simple cadre territorial anatolien signifie désormais aussi faire partie de la famille turque, les deux appartenances se conditionnant mutuellement. Aussi la rupture avec le monde musulman devient-elle la condition même d'être musulman en Turquie, le «Turc » étant clairement défini comme «celui qui partage la même langue et la même religion »(33). Accepter l'islam dans la nouvelle République revient dès lors à accepter la turcité et inversement, "sortir» de la turcité devient synonyme de quitter l'islam, une sorte de «khurudj», qui est signe de trahison (34).

L'État se charge de la défense de cette famille qu'est la nation turque, et dans son islamité, qui la distingue de l'Occident chrétien, et dans sa turcité, qui la sépare du monde musulman. Ainsi, c'est dans une configuration toute nouvelle, explicitement anti-ottomane (et non pas simplement post-ottomane), que la politique de fonctionnarisation des religieux est maintenue. Dès le 2 mars 1924, une présidence aux Affaires religieuses, dépendant directement du Premier ministre, est créée.

(30) Les alévis, qui constituent quelque $20 \%$ de la population et dont le dogme se distingue radicalement de l'islam sunnite et chi'ite, sont purement et simplement assimilés aux musulmans sunnites. Cf. pour cette communauté-confession, Paul J. White, Joost Jongerden, dir., Turkey's Alevi Enigma. A Comprehensive Overwiev, Leyde, Brill, 2003.

(31) Cf. Taner AксAм, Türk Ulusal Kimligi ve Ermeni Sorunu, Istanbul, Iletisim Yayinlari, 1992.

(32) Dogan Duman, Demokrasi Sürecinde Türkiye'de Islamcilik, Izmir, Dokuz Eylül Yayinlari, 1999 , p. 23.

(33) Halil NimetüL (1928) cité par Ibrahim BAHAdir, Ümmetten Millete. Türk Ulusunun Insasi, 1860-1945, Ankara, Kalan Yayinlari, 2001, p. 149.

(34) En effet, comme le rappelle Ghassan Salamé : "Contester c'était sortir, sortir c'était trahir », Ghassan Salamé, " Où sont donc les démocrates ", in Ghassan SAlamé, dir., Démocraties sans démocrates. Politiques d'ouverture dans le monde arabe et islamique, Paris, Fayard, 1994, p. 23. 
L'organisation de l'islam comme élément fondateur et central de la nation (35), devient d'ailleurs une prérogative régalienne, toute instance non fonctionnarisée, dont les confréries religieuses, étant interdites dès 1925, toute expression de la religiosité, qui échappe au contrôle de l'État, ou qui n'émane pas de lui, étant rudement sanctionnée (36). En effet, comme le suggère M. A. Agaogullari : « l'État, d'abord par le biais de la Présidence des Affaires religieuses, puis $\{\ldots\}$, par celui de la Direction des Fondations religieuses, institue une surveillance étroite sur le corps religieux. Ce dernier appartenant au statut de fonctionnaire, les religieux réfractaires tombent sous le coup des sanctions administratives et peuvent être écartés facilement de leurs fonctions. L'État se donne aussi les moyens juridiques pour réprimer les religieux qui ne respectent pas les mesures laïques » (37).

Mais, en même temps, toute activité missionnaire, susceptible de convertir les musulmans, à savoir, les Turcs, au christianisme, donc de les pervertir et transformer en «intrus » dans la famille turque, est tout aussi lourdement pénalisée. Ainsi, la conversion, dans les années 1930, de plusieurs jeunes lycéennes au christianisme provoque une crise majeure, car elle est interprétée comme une sortie de la nation turque. Les jeunes filles, expulsées de leur lycée, sont considérées, ni plus ni moins comme des « traîtresses », coupables de l'intrusion d'une autre religion dans un corps national qui exige pourtant l'homogénéité religieuse (38). De même, les traités internationaux, notamment ceux de Lausanne (1923) aidant, les membres des minorités arménienne, grecque et juive, autrement dit des communautés chrétiennes, notamment balkaniques, mais aussi, les Grecs et Arméniens d'Anatolie, sont considérés comme citoyens turcs, sans pour autant faire partie de la nation turque, laquelle est définie par l'appartenance à l'islam. À l'inverse, les communautés musulmanes non-turques, telle que la communauté kurde, sont assimilées à la turcité - entre autres - en raison de leur appartenance à l'islam.

Quel sens donner alors à la laïcité qui est introduite dès 1937 comme l'une des six flèches officielles de la nation turque dans la Constitution (39) qui fait suite à la suppression de l'article concernant la religion d'État en 1928, et aux mesures draconiennes prises à l'encontre de la religion en tant que telle comme la fermeture des mosquées ou leur transformation en écurie ? Comment concevoir d'ailleurs la laïcité alors même que le nationalisme et l'islam constituent l'«expression of a single underlying ideology » (40) dans ce pays ? Les textes fondateurs de Mustafa

(35) Pour de multiples initiatives officielles et les textes de loi visant à organiser l'islam comme une religion à la fois rationnelle et nationale, cf. Gotthard Jäschke, Yeni Türkiye'de Islâmlik, Ankara, Bilgi Yayinevi, 1972.

(36) Pour ces mouvements, qui ne se réduisent naturellement pas à leur seul trait religieux, cf. Gavin D. BRocketT, «Collective Action and the Turkish Revolution: Towards a Framework for the Social History of the Atatürk Era, 1923-1938 », in Sylvia Kedouri, dir., Turkey Before and After Atatürk. Internal and External Affairs, Londres, Frank Cass, 1999, pp. 44-66.

(37) Mehmet Ali Agaogullari, L'islam dans la vie politique de la Turquie, Ankara, Faculté des Sciences politique de l'Université d'Ankara, 1982, p. 52.

(38) «La propagande chrétienne » reste d'ailleurs un délit en Turquie. Cf. Çetin ÖzEK, Devlet ve Din, Istanbul, Ada Yayinlari, s.d., p. 495.

(39) Les autres « flèches » du Parti Républicain du Peuple qui devient à cette date Parti-État, sont : le nationalisme, le républicanisme, le populisme, l'étatisme et le révolutionnarisme. Ensemble avec le laïcisme, ces «Flèches » constituent également la base des constitutions successives de la République de Turquie, dont la dernière, en vigueur depuis 1982.

(40) Richard TAPPER, Nancy TAPPER, « Religion, Education and Continuity in a Provincial Town », in Richard TAPPER, dir., Islam in Modern Turkey. Religion, Politics and Literature in a Secular State, Londres, I.B. Tauris, 1991, p. 80. 
Kemal sur la laïcité apportent une réponse on ne peut plus claire à cette question et donnent un sens aux énoncés contradictoires du nouveau pouvoir : " puisque, Dieu Merci nous sommes tous Musulmans, nous pouvons et nous devons être tous laïques »(41). La laïcité ne signifie donc pas l'autonomisation mutuelle des champs religieux et politique, encore moins la neutralité de l'État dans le domaine de la conscience et des pratiques du culte. Bien au contraire, l'islamité de la nation est posée comme condition sine qua non de son passage à la laïcité. Le conflit autour de la religion, et partant, autour de la nation, n'oppose donc pas les courants qui ont différentes définitions de la place de la religion dans la société : il oppose les membres d'une même famille qui, enfin débarrassée de l'« autre » religieusement déterminé, cherchent à redéfinir les frontières entre le licite et l'illicite, entre la loyauté et l' " exit», entre les formes d'expression de la religion dans l'espace privé - intime - et dans l'espace commun.

Le discours sur la laïcité, qui comme le rappelle Olivier Abel, est détaché de tout souci de garantir la pluralité des convictions religieuses (42), devient ainsi un instrument du pouvoir et fait, avec le nationalisme, partie de la doxa républicaine. Construite sur fonds de mesures drastiques et des pratiques par moment très coercitives - comme l'interdiction des confréries et les exécutions massives des opposants à "la réforme de chapeau » en 1925 - (43) ou encore de la répression qui suit un incident de nature messianique en 1930 (44), cette doxa substitue les mots d'ordre du pouvoir à toute réflexion sur les contradictions du nouveau régime kémaliste. Celles-ci sont pourtant indéniables : légitimer la nation comme musulmane, donc turque, intégrer par conséquent l'islamité comme condition d'appartenance à la turcité, justifier la gestion directe du domaine religieux par l'État; supprimer donc toute autonomie interne de la sphère religieuse subordonnée, et fixer en même temps la laïcité comme l'objectif non négociable de la nation. Derrière la doxa laïque de la République, il y a bien sûr aussi la question de savoir qui va exercer l'autorité politique, et partant l'autorité tout court au nom de la nation. À ce propos Mustafa Kemal tranche : «Si les masses sont restées sous l'influence des traditions asiatiques, les intellectuels qui représentent la souveraineté nationale au nom de ces masses, passent à l'action. Pour leur procurer un

(41) Comme le dit Taha Parla, «Lorsque l'on additionne ces \{citations\} nous n'obtenons pas (et nous ne pourrons pas obtenir) une laïcité comprise comme une séparation totale de la religion et du politique, de l'État et de la religion et des affaires de l'État et d'ici-bas. La laïcité dans un sens plus étroit signifie que les «autres » ne puissent pas mêler la religion à la politique, mais que le chef et le parti unique, qui connaissent ce qui est mieux, puissent mettre la religion sous l'ordre de l'État ", Taha PARLA, Türkiye'de Siyasal Kültürün Resmi Kaynaklari, v.3, Kemalist Tek-Parti Ideolojisi ve CHP'nin Alti Oku, Istanbul, Iletisim, 1992, p. 278. Pour les textes juridiques de la laïcité en Turquie, cf. Gérard Groc, «Textes officiels turcs sur la laïcité », CEMOTI, n 19, 1995, pp. 313-332.

(42) Olivier ABEL, «Que veut dire la laïcité ? », CEMOTI, nº 10, 1990. Cf. aussi ses remarques : «Le problème se pose donc $\{\ldots\}$ dans la mesure où une part de plus en plus importante des intellectuels et des responsables turcs se disent qu'on ne peut imposer la laïcité de l'extérieur, sans qu'il y ait une structure d'accueil interne aux religions qui rende la greffe laïque possible. Cette structure théologique suppose une critique interne des religions qui n'est possible que dans un contexte de pluralisme religieux. Or ce pluralisme, et par là cette critique idéologique interne, sont bloqués par la structure même de la laïcité turque », Olivier ABEL, « La condition laïque. Réflexions sur le problème de la laïcité en Turquie et en France », CEMOTI, no 19, 1995, p. 43.

(43) Cf. pour une partie des actes, Ahmed Nedim, ed., Ankara Istiklâl Mahkemesi Zabitlari 1926, Istanbul Isaret Yayinlari, 1993.

(44) Cf. Hamit Bozarslan, «Le mahdisme en Turquie : 1"“incident de Menemen" en 1930», REMMM, no 91-94, 2001, pp. 297-320. 
système moderne, ils combattent le système arriéré, les fausses croyances, les superstitions. Ils font la révolution. Ils changent le système. Pour cela, on ne recourt pas au plébiscite » (45).

Le discours (et la pratique) sur la laïcité doivent dès lors construire et sublimer l'islam comme la dernière, donc la seule vraie religion, et partant disqualifier les autres religions révélées, et en même temps, assurer la mainmise de l'État sur le domaine religieux (46) et interdire tout signe de visibilité de l'islam en tant que religion dans l'espace public. La religion dont l'État devient le seul gestionnaire, est confinée à un espace clos et condamnée à l'invisibilité. Elle est certes un élément de cohésion de la nation et le pouvoir n'hésite pas à recourir à l'impératif coranique pour obtenir l'obéissance de ses sujets (47). Mais l'essence et la finalité de la nation sont à chercher ailleurs, dans le registre de la turcité. Or, cette turcité se trouve également, et inextricablement, liée à un autre impératif qui est l'occidentalisation. L'occidentalisme ne signifie surtout pas devenir une partie de l'Occident, autrement dit, se supprimer pour devenir l'autre, perspective à laquelle répugnent les élites kémalistes, mais simplement, s'approprier les éléments de supériorité qui font que l'autre est fort et puissant. S'opposer à cet objectif c'est accepter d'affaiblir la nation turque, qui est musulmane, en même temps que la priver de la religion qui lui permet de rester distincte de l'autre qu'elle veut bien imiter. Partant, l'islamisme, comme le khurudj, devient synonyme de trahison et de fitna.

En somme la politique kémaliste peut être résumée comme un double programme. En premier lieu, il s'agit de créer un islam rationnel et national, chargé de légitimer le pouvoir, en cautionnant sa volonté d'éradiquer, au nom du progrès, de la révolution et de la civilisation, toute religiosité non officielle (confrérique, « ottomane », « arabe», populaire...). La religion et le religieux seront donc soumis ou ne seront pas. En deuxième lieu, dans une société dont la légitimité réside dans l'homogénéité religieuse, la laïcité est érigée en obligation constitutionnelle, condition de la citoyenneté même. La nation dont l'islamité est préalablement admise, sera laïque ou ne sera pas.

Comment s'étonner dès lors que tout débat autour de la religion en Turquie renvoie plus aux enjeux politiques et nationaux qu'à la question de la laïcité proprement dite? Et comment s'étonner que dans ce système qui impose d'emblée l'appartenance à une religion comme condition d'accès à la laïcité, la subordination des religieux au pouvoir par la fonctionnarisation, leur assure un emploi à vie, mais ne les met pas à l'abri du statut d'ennemis de l'intérieur, réels ou potentiels, de la République?

Hamit BOZARSLAN

Institut d'études de l'islam et des sociétés du monde musulman-EHESS

(45) Cité par Mehmet A. Agaogullari, op.cit., p. 83.

(46) Ce qui est vu comme la seule garantie dont dispose la République contre les dérapages dont l'islam est sensé être porteur. Cf. à ce propos, l'ouvrage de l'un des plus importants défenseurs de la laïcité kémaliste, Nur SERTER, Dinde Siyasal Islam Tekeli, Istanbul, Sarmal Yayinevi, 1997.

(47) Faruk BILICI, «Islam, modernité et éducation religieuse en Turquie » in Semih VANER, Modernisation autoritaire en Turquie et en Iran, Paris, L'Harmattan, 1991, pp. 41-60. 
Résumé

Cet article porte sur les rapports entre la religion et la laïcité comme deux répertoires de légitimation de l'autorité, et partant, du pouvoir politique, en Turquie. Partant d'une perspective de la longue durée, il souligne qu'alors que l'islam se confond avec la nation et est présenté comme l'un des garants de son unité, voire de son unanimisme, la laïcité, mot fétiche qui résume toute la problématique de l'occidentalisation, s'avère être l'une des perpétuelles sources de sa division. Cette contradiction aboutit une autre, potentiellement bien plus violente: soumis ou non au pouvoir, le religieux est toujours conçu comme l'ennemi, réel ou potentiel, de la nation, laquelle ne peut pourtant exister sans la religion.

\section{Abstract}

This article analysis the relations between religion and laicism, which are presented as two complementary repertoires of the authority, and therefore, the political power, in Turkey. In the framework of a long-term historical perspective, it suggests that, on the one hand, Islam is presented as a decisive element of the national identity and as one of the warrants of the national unity; on the other hand, laicism, which summarises countries long-going westernization "problématique" becomes a constant source of division. The tension between these two terms gives birth to a potentially more violent contradiction: while the nation cannot exist without its intrinsic relation with the religion, the religious establishment is considered as the nations real or would-be enemy.

\section{Resumen}

Este artículo versa sobre las relaciones entre la religión y la laicidad como dos principios de legitimación de la autoridad, a partir del poder politico, en Turquia. Partiendo de una perspectiva de larga duración, el artículo destaca que, mientras que el Islam se confunde con la nación, y es presentado como uno de los garantes de su unidad, incluso de su unanimidad, la laicidad, concepto fetiche que resume toda la problemática de la occidentalización, se revela como una de las fuentes permanentes de su división. Esta contradicción lleva a otra, potencialmente mucho más violenta: sometido o no al poder, lo religioso es siempre concebido como el enemigo, real o potencial, de la nación, la que no puede, sin embargo, existir sin la religión. 
